\title{
Strong list-chromatic index of subcubic graphs
}

\author{
Tianjiao Dai ${ }^{1 *}, \quad$ Guanghui Wang ${ }^{1 \dagger}, \quad$ Donglei Yang ${ }^{1 \ddagger}$ Gexin $\mathrm{Yu}^{2,3 \S}$ \\ ${ }^{1}$ Department of Mathematics, Shandong University, Jinan, Shandong, China. \\ ${ }^{2}$ Department of Mathematics, The College of William and Mary, Williamsburg, VA, USA. \\ ${ }^{3}$ Department of Mathematics, Central China Normal University, Wuhan, Hubei, China.
}

\begin{abstract}
A strong $k$-edge-coloring of a graph $\mathrm{G}$ is an edge-coloring with $k$ colors in which every color class is an induced matching. The strong chromatic index of $G$, denoted by $\chi_{s}^{\prime}(G)$, is the minimum $k$ for which $G$ has a strong $k$-edge-coloring. In 1985, Erdős and Nešetřil conjectured that $\chi_{s}^{\prime}(G) \leq \frac{5}{4} \Delta(G)^{2}$, where $\Delta(G)$ is the maximum degree of $G$. When $G$ is a graph with maximum degree at most 3 , the conjecture was verified independently by Andersen and Horák, Qing, and Trotter. In this paper, we consider the list version of strong edge-coloring. In particular, we show that every subcubic graph has strong list-chromatic index at most 11 and every planar subcubic graph has strong list-chromatic index at most 10.
\end{abstract}

Keywords: Subcubic graphs; Strong list-chromatic index; Combinatorial Nullstellensatz

\section{Introduction}

All graphs in this paper are finite and simple. A strong $k$-edge-coloring of a graph $G$ is a coloring $\phi: E(G) \longrightarrow[k]$ such that if any two edges $e_{1}$ and $e_{2}$ are either adjacent to each other or adjacent to a common edge, then $\phi\left(e_{1}\right) \neq \phi\left(e_{2}\right)$. In other words, the edges in each color class give an induced matching in the graph; that is, any two vertices belonging to distinct edges with the same color are not adjacent. The strong chromatic index of $G$, denoted by $\chi_{s}^{\prime}(G)$, is the minimum $k$ for which $G$ has a strong $k$-edge-coloring.

The following conjecture was proposed by Erdős and Nešetřil in 1985 at Prague.

Conjecture 1.1 [6, 7] If $G$ is a graph with maximum degree $\Delta(G)$, then

$$
\chi_{s}^{\prime}(G) \leq \begin{cases}\frac{5}{4} \Delta(G)^{2}, & \text { if } \Delta(G) \text { is even, } \\ \frac{5}{4} \Delta(G)^{2}-\frac{1}{2} \Delta(G)+\frac{1}{4}, & \text { if } \Delta(G) \text { is odd } .\end{cases}
$$

\footnotetext{
*Email: dtjmath@163.com.

${ }^{\dagger}$ Corresponding author. Email: ghwang@sdu.edu.cn. Supported by the National Natural Science Foundation of China (11471193,11631014), the Foundation for Distinguished Young Scholars of Shandong Province (JQ201501).

‡Email: yangdonglei_sdu@163.com.

${ }^{\S}$ Email: gyu@wm.edu. Supported by the NSA (H98230-16-1-0316) and NSFC (11728102).
} 
Note that there are examples showing that the conjectured upper bound is tight (i.e. blowups of a 5-cycle). Andersen [2] and independently Horák, Qing, and Trotter [9] showed that $\chi_{s}^{\prime}(G) \leq 10$ for any graph $G$ with $\Delta(G)=3$, thus settling the first nontrivial case of Conjecture 1.1. Cranston [5] gave an algorithm that uses at most 22 colors for every graph with $\Delta(G)=4$, which was improved to 21 very recently by Huang, Santana and Yu [16]. When $\Delta(G)$ is sufficiently large, Molloy and Reed [15] proved that $\chi_{s}^{\prime}(G) \leq 1.998 \Delta(G)^{2}$. Henning Bruhn and Felix Joos [4] showed that $\chi_{s}^{\prime}(G) \leq 1.93 \Delta(G)^{2}$. Recently, Bonamy, Perrett, and Postle [3] improved the upper bound to $1.835 \Delta(G)^{2}$.

In this article, we study the list version of strong edge-coloring. For each $e \in E(G)$, let $L(e)$ be the list of available colors of $e$, and let $L=\{L(e): e \in E(G)\}$. The graph $G$ is strongly L-edgecolorable if there exists a strong edge coloring $c$ of $G$ such that $c(e) \in L(e)$ for every $e \in E(G)$. For a positive integer $k$, a graph $G$ is strongly $k$-edge-choosable if $G$ is strongly $L$-edge colorable for every $L$ with $|L(e)| \geq k$ for all $e \in E(G)$. The strong list-chromatic index, denoted by $\chi_{s, l}^{\prime}(G)$, is the minimum positive integer $k$ for which $G$ is strongly $k$-edge-choosable. Note that $\chi_{s}^{\prime}(G) \leq \chi_{s, l}^{\prime}(G)$ for every graph $G$.

The probablistic arguments that Molloy-Reed and Bonamy-Perrett-Postle used to give upper bounds of $\chi_{s}^{\prime}$ on graphs of large $\Delta(G)$ actually also work for the strong list-chromatic index. So we have $\chi_{s, l}^{\prime}(G) \leq 1.835 \Delta(G)^{2}$ for large $\Delta(G)$. Ma, Miao, Zhu, Zhang and Luo [14] proved that the strong list-chromatic index of a subcubic graph with maximum average degree less than $\frac{15}{7}, \frac{27}{11}, \frac{13}{5}, \frac{36}{13}$ is at most $6,7,8,9$, respectively. More results of this kind can be found in [17].

In this paper, we prove the following result.

Theorem 1.2 If $\Delta(G) \leq 3$, then $\chi_{s, l}^{\prime}(G) \leq 11$.

For planar graphs, we actually can do a little better.

Theorem 1.3 If $G$ is a subcubic planar graph, then $\chi_{s, l}^{\prime}(G) \leq 10$.

Recall that Andersen [2] and Horák, Qing, and Trotter [9] proved that $\chi_{s}^{\prime}(G) \leq 10$ if $\Delta(G) \leq 3$. Kostochka et. al. [12] proved that $\chi_{s}^{\prime}(G) \leq 9$ under the additional assumption that $G$ is planar. We do not feel that our results are optimal, but it may involve substantial work to improve them. One of the main tools we use is Hall's Theorem.

Lemma 1.4 (Hall [8]) Let $A_{1}, \ldots, A_{n}$ be $n$ subsets of a set $U$. Distinct representatives of $\left\{A_{1}, \ldots, A_{n}\right\}$ exist if and only if for all $k, 1 \leq k \leq n$ and every choice of subcollection of size $k,\left\{A_{i_{1}}, \ldots, A_{i_{k}}\right\}$, we have $\left|A_{i_{1}} \cup \ldots \cup A_{i_{k}}\right| \geq k$. 
Another tool we use is the Combinatorial Nullstellensatz.

Lemma 1.5 (Alon [1], Combinatorial Nullstellensatz) Let $\mathbb{F}$ be an arbitrary field, and let $P=$ $P\left(x_{1}, x_{2}, \ldots, x_{n}\right)$ be a polynomial in $\mathbb{F}\left[x_{1}, x_{2}, \ldots, x_{n}\right]$. Suppose that the degree deg $(P)$ of $P$ equals $\sum_{i=1}^{n} k_{i}$, where each $k_{i}$ is a non-negative integer, and the coefficient of $x_{1}^{k_{1}} x_{2}^{k_{2}} \cdots x_{n}^{k_{n}}$ in $P$ is nonzero. Then if $S_{1}, S_{2}, \ldots, S_{n}$ are subsets of $\mathbb{F}$ with $\left|S_{i}\right|>k_{i}, i=1,2, \ldots, n$, there exist $s_{1} \in S_{1}, s_{2} \in$ $S_{2}, \ldots, s_{n} \in S_{n}$ so that $P\left(s_{1}, s_{2}, \ldots, s_{n}\right) \neq 0$.

We use MATLAB to calculate the coefficients of specific monomials. Let $P=P\left(x_{1}, x_{2}, \ldots, x_{n}\right)$ be a polynomial in $n$ variables, where $n \geq 1$. By $c_{p}\left(x_{1}^{k_{1}} x_{2}^{k_{2}} \cdots x_{n}^{k_{n}}\right)$, we denote the coefficient of the monomial $x_{1}^{k_{1}} x_{2}^{k_{2}} \cdots x_{n}^{k_{n}}$ in $P$, where $k_{i}(1 \leq i \leq n)$ is a non-negative integer. The codes are listed in the Appendix.

\section{Basic properties}

Consider $(G, L)$ such that $G$ is not $L$-choosable but any proper subgraph of $G$ is $L$-choosable. Clearly, $G$ is connected. In this section, we will show that if $|L(e)| \geq 10$ for each $e \in E(G)$, then $G$ is cubic and has no cycles of length at most five.

We first introduce some notation. An $i$-vertex is a vertex of degree $i$ in our graphs. An $i$-cycles is a cycle of length $i$ in graphs. A partial coloring of $G$ is a coloring of a proper subgraph of $G$. Given edges $e$ and $e^{\prime}$ in $G$, we say that $e$ sees $e^{\prime}$ if either $e$ and $e^{\prime}$ are adjacent, or there is another edge $e^{\prime \prime}$ adjacent to both $e$ and $e^{\prime}$. Note that even if $e$ sees $e^{\prime}$ in $\mathrm{G}, e$ does not necessarily see $e^{\prime}$ in a proper subgraph of $G$. Additionally, we will also say that $e$ sees a color $\alpha$ if $e$ sees an edge $e^{\prime}$ of color $\alpha$. Let $\phi$ be a partial coloring of $G$. For $e \in E(G)$, let $C_{\phi}(e)$ denote the set of colors seen by $e$, and let $A_{\phi}(e)=L(e) \backslash C_{\phi}(e)$. For $v \in V(G), H \subseteq G$, let $d(v, H)$ with respect to $v$ be the minimum of the lengths of the $u-v$ paths of $G$ where $u \in V(H)$.

Lemma 2.1 $G$ is cubic.

Proof. By way of contradiction, we assume that $d(v) \leq 2$ for some $v \in V(G)$. By the minimality of $G, G-v$ has an $L$-coloring $\phi$. First let $v$ be a 1 -vertex incident with the edge $e$. Since $\left|C_{\phi}(e)\right| \leq 6$, $\left|A_{\phi}(e)\right| \geq 4$, so $e$ can be colored. Let $v$ be a 2 -vertex with incident edges $e_{1}$ and $e_{2}$. Since $\left|C_{\phi}\left(e_{i}\right)\right| \leq 8$ for $i=1,2,\left|A_{\phi}\left(e_{i}\right)\right| \geq 2$. So we can color $e_{1}$ and $e_{2}$ in any order.

Lemma 2.2 G has no triangles. 
Proof. Suppose that $G$ contains a triangle: $v_{1} v_{2} v_{3} v_{1}$ (see Fig.1 (1)). By the minimality of $G$, let $\phi$ be an $L$-coloring of the subgraph $H=G-v_{1}$. Note that $\left|A_{\phi}\left(e_{i}\right)\right| \geq 3$, for $i=1,2$ and $\left|A_{\phi}\left(e_{3}\right)\right| \geq 1$. Then $\phi$ can be extended to an $L$-coloring of $G$ by Lemma 1.4 .

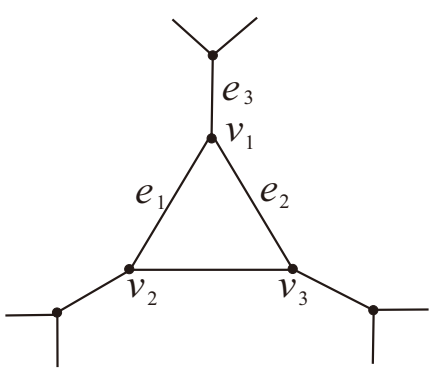

(1)

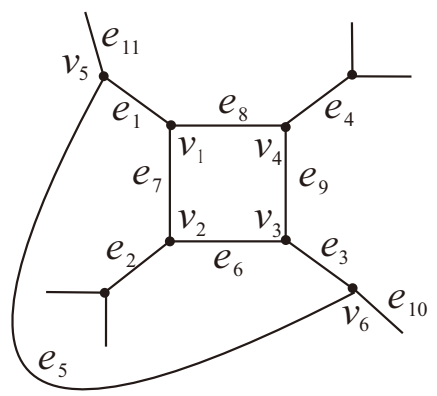

(4)

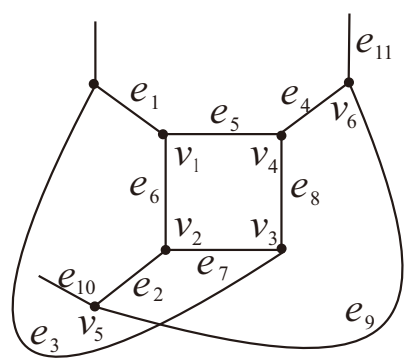

(7)

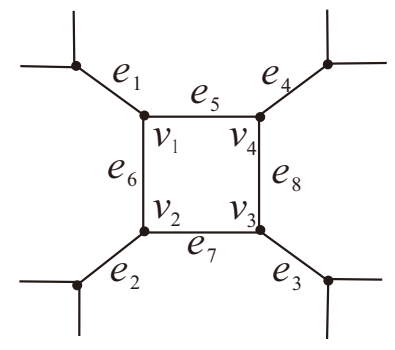

(2)

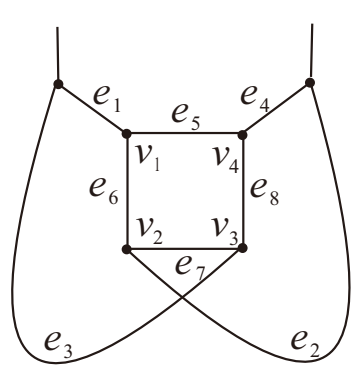

(5)

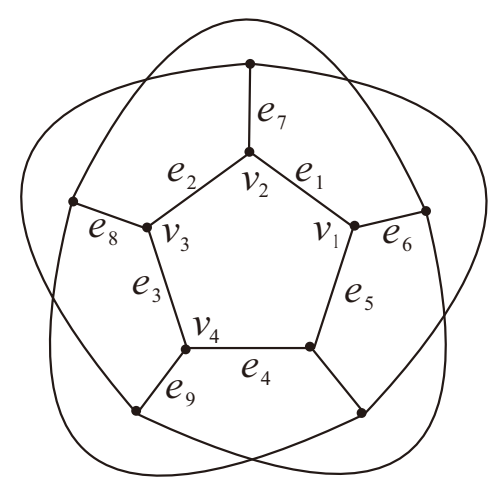

(8)

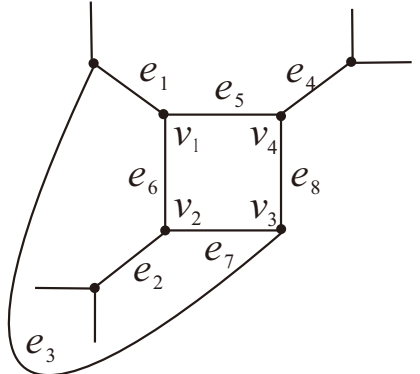

(3)

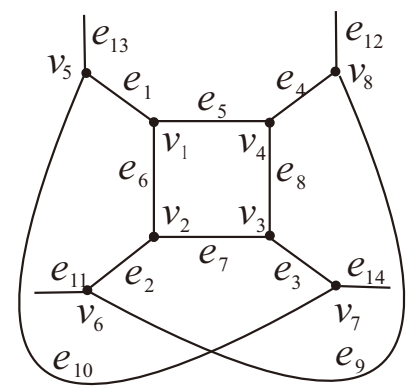

(6)

Figure 1: Structures with 3-, 4-, and 5-cycles

Lemma 2.3 G has no 4-cycles.

Proof. Suppose that $G$ contains a 4-cycle. By Lemma 2.2, each 4-cycle must be an induced 4-cycle, and we divide all 4-cycles into three classes. The possible local structures about a 4-cycle are shown in Figure $1(2)-(7)$. For $2 \leq i \leq 7$, let $H_{i}$ be the subgraph of $G$ obtained by removing 
the vertices with labels in Figure 1 (i) for $2 \leq i \leq 7$. By minimality of $G, H_{i}$ has an $L$-coloring $\phi$. Let $A_{i}=A_{\phi}\left(e_{i}\right)$ for each labelled edge $e_{i}$ in the figures.

When $e_{1}$ does not see $e_{3}$ and $e_{2}$ does not see $e_{4}$, we consider $H_{2}$.

In $H_{2}$, we have $\left|A_{i}\right| \geq 4$ for $i=1,2,3,4$ and $\left|A_{i}\right| \geq 6$ for $i=5,6,7,8$. By Lemma 1.4, we may assume that for some $I \subseteq[8],\left|\bigcup_{i \in I} A_{i}\right|<|I|$. So $|I|>6$, and $|I| \in\{7,8\}$. By symmetry, let $1,3 \in I$. Then $\left|A_{1} \cup A_{3}\right|<8$, so there exists $\alpha \in A_{1} \cap A_{3}$, and we color $e_{1}$ and $e_{3}$ with $\alpha$. Let $A_{i}^{\prime}=A_{i}-\{\alpha\}$. Now for $J \subseteq[8]-\{1,3\}$, if $\left|\bigcup_{i \in J} A_{i}^{\prime}\right|<|J|$, then $|J|>3$, which implies that $J \cap\{5,6,7,8\} \neq \emptyset$, so $|J| \geq 6$. Then $|J|=6,\left|A_{i}^{\prime}\right| \geq 5$ for $i \in\{5,6,7,8\}$ and $A_{2}^{\prime} \cap A_{4}^{\prime} \neq \emptyset$. Color $e_{2}$ and $e_{4}$ with $\beta \in A_{2}^{\prime} \cap A_{4}^{\prime}$, then we can color $e_{5}, e_{6}, e_{7}, e_{8}$ in any order, a contradiction.

When $e_{1}$ sees $e_{3}$ and $e_{2}$ does not see $e_{4}$, we consider $H_{3}$ and $H_{4}$.

Consider $H_{3}$. Note that $\left|A_{i}\right| \geq 7$ for $i=1,3,5,6,7,8$ and $\left|A_{i}\right| \geq 4$ for $i=2,4$. By Lemma 1.4 . we may assume that for some $I \subseteq[8],\left|\bigcup_{i \in I} A_{i}\right|<|I|$. Then $I=[8],\left|A_{i}\right| \geq 7$ for $i \in\{1,3,5,6,7,8\}$ and $A_{2} \cap A_{4} \neq \emptyset$. Color $e_{2}$ and $e_{4}$ with $\alpha \in A_{2} \cap A_{4}$, and then we can color the rest of edges one by one, a contradiction.

Consider $H_{4}$. Note that $\left|A_{i}\right| \geq 4$ for $i=2,4,10,11,\left|A_{5}\right| \geq 6$ and $\left|A_{i}\right| \geq 8$ for $i \in\{1,3,6,7,8,9\}$. By Lemma 1.4, we may assume that for some $I \subseteq[11],\left|\bigcup_{i \in I} A_{i}\right|<|I|$. Then $\mid I \cap\{1,3,6,7,8,9\} \neq \emptyset$, so $|I| \geq 9$.

We consider the following cases.

Case 1: $\{6,11\} \subset I$ (or by symmetry, $\{7,10\} \subset I$ ). Then $A_{6} \cap A_{11} \neq \emptyset$ and color $e_{6}$ and $e_{11}$ with $\alpha \in A_{6} \cap A_{11}$. For $i \in[11]-\{6,11\}$, let $A_{i}^{\prime}=A_{i}-\{\alpha\}$. Then for some $J \subseteq[11]-\{6,11\}$, $\left|\bigcup_{i \in J} A_{i}^{\prime}\right|<|J|$. Then $|J| \geq 8$. So at most one of $2,4,5,7,10$ is not in $J$.

- $5 \in J$. We may assume that $2 \in J$ as well (or by symmetry, $4 \in J$ ). Then $A_{2}^{\prime} \cap A_{5}^{\prime} \neq \emptyset$, and we color $e_{2}$ and $e_{5}$ with $\beta \in A_{2}^{\prime} \cap A_{5}^{\prime}$. Let $A_{i}^{\prime \prime}=A_{i}-\{\alpha, \beta\}$ for $i \in[11]-\{2,5,6,11\}$. Then for some $K \subseteq[11]-\{2,5,6,11\},\left|\bigcup_{i \in K} A_{i}^{\prime \prime}\right|<|K|$. So $K \cap\{1,3,7,8,9\} \neq \emptyset$ and thus $|K|=7$. Now we can color $e_{7}$ and $e_{10}$ with $\gamma \in A_{7}^{\prime \prime} \cap A_{10}^{\prime \prime}$, and by Lemma 1.4 , color the rest of the edges.

- $5 \notin J$. Then $|J|=8$, so $A_{2}^{\prime} \cap A_{4}^{\prime} \neq \emptyset$. Color $e_{2}$ and $e_{4}$ with $\beta \in A_{2}^{\prime} \cap A_{4}^{\prime}$. Let $A_{i}^{\prime \prime}=A_{i}-\{\alpha, \beta\}$ for $i \in[11]-\{6,11,2,4\}$. Then for some $K \subseteq[11]-\{2,4,6,11\},\left|\bigcup_{i \in K} A_{i}^{\prime \prime}\right|<|K|$. So $K \cap\{1,3,7,8,9\} \neq \emptyset$ and thus $|K|=7$. Now we can color $e_{7}$ and $e_{10}$ with $\gamma \in A_{7}^{\prime \prime} \cap A_{10}^{\prime \prime}$, and by Lemma 1.4 , color the rest of the edges.

Case 2: $\{6,11\} \not \subset I$ and $\{7,10\} \not \subset I$. Then $|I|=9$ and $2,4,5 \in I$. So $A_{2} \cap A_{5} \neq \emptyset$. Color $e_{2}$ and $e_{5}$ with $\alpha \in A_{2} \cap A_{5}$. For $i \in[11]-\{2,5\}$, let $A_{i}^{\prime}=A_{i}-\{\alpha\}$. Then for some $J \subseteq[11]-\{2,5\}$, $\left|\bigcup_{i \in J} A_{i}^{\prime}\right|<|J|$. Then $|J| \geq 8$. Then $\{6,11\} \subset J$ (or by symmetry $\{7,10\} \subset J$ ), and $A_{6} \cap A_{11} \neq \emptyset$ 
and color $e_{6}$ and $e_{11}$ with $\beta \in A_{6} \cap A_{11}$. Let $A_{i}^{\prime \prime}=A_{i}-\{\alpha, \beta\}$ for $i \in[11]-\{2,5,6,11\}$. Then for some $K \subseteq[11]-\{2,5,6,11\},\left|\bigcup_{i \in K} A_{i}^{\prime \prime}\right|<|K|$. So $K \cap\{1,3,7,8,9\} \neq \emptyset$ and thus $|K|=7$. Now we can color $e_{7}$ and $e_{10}$ with $\gamma \in A_{7}^{\prime \prime} \cap A_{10}^{\prime \prime}$, and by Lemma 1.4 , color the rest of the edges.

When $e_{1}$ sees $e_{3}$ and $e_{2}$ sees $e_{4}$, we consider $H_{5}, H_{6}$ and $H_{7}$.

Consider $H_{5}$. Note that $\left|A_{i}\right| \geq 7$ for $i=1,2,3,4$ and $\left|A_{i}\right| \geq 8$ for $i=5,6,7,8$. By Lemma 1.4 . we may assume that for some $I \subseteq[8],\left|\bigcup_{i \in I} A_{i}\right|<|I|$. Clearly, no such $I$ exists, a contradiction.

Consider $H_{6}$. First, $\left|A_{\phi}\left(e_{i}\right)\right| \geq 4$ for $i=11,12,13,14$. We can make it that the colors of $e_{i}$ are different by Lemma 1.4 for $i=11,12,13,14$. Then we note that $\left|A_{i}\right| \geq 6$ for $i=1,2,3,4,\left|A_{i}\right| \geq 8$ for $i=5,6,7,8$ and $\left|A_{i}\right| \geq 4$ for $i=9,10$. By Lemma 1.4, we may assume that for some $I \subseteq[10]$, $\left|\bigcup_{i \in I} A_{i}\right|<|I|$. Then $|I|>8$, so $|I| \in\{9,10\}$. By symmetry, we may assume that $\{4,10\} \subset I$. Then $A_{4} \cap A_{10} \neq \emptyset$, so color $e_{4}, e_{10}$ with $\alpha \in A_{4} \cap A_{10}$. Let $A_{i}^{\prime}=A_{i}-\{\alpha\}$ for $i \in[10]-\{4,10\}$. Then for some $J \subseteq[10]-\{4,10\},\left|\bigcup_{i \in J} A_{i}^{\prime}\right|<|J|$. It implies that $J \cap\{5,6,7,8\} \neq \emptyset$, thus $J=[10]-\{4,10\}$, and $\left|A_{i}^{\prime}\right| \geq 7$ for $i \in\{5,6,7,8\}$ and $A_{1}^{\prime} \cap A_{9}^{\prime} \neq \emptyset$. Color $e_{1}$ and $e_{9}$ with $\beta \in A_{1}^{\prime} \cap A_{9}^{\prime}$, then we can color the rest of the edges one by one, a contradiction.

Consider $H_{7}$. First, $\left|A_{\phi}\left(e_{i}\right)\right| \geq 4$ for $i=10,11$. We can make it that the color of $e_{10}$ is different from the color of $e_{11}$. Then we note that $\left|A_{i}\right| \geq 7$ for $i=1,3,\left|A_{i}\right| \geq 6$ for $i=2,4,\left|A_{i}\right| \geq 8$ for $i=5,6,7,8$ and $\left|A_{\phi}\left(e_{9}\right)\right| \geq 4$. By Lemma 1.4 , we may assume that for some $I \subseteq[9],\left|\bigcup_{i \in I} A_{i}\right|<|I|$. Then $I=[9]$ and $A_{3} \cap A_{9} \neq \emptyset$. Color $e_{3}$ and $e_{9}$ with $\alpha \in A_{3} \cap A_{9}$, and then we can color the rest of edges one by one, a contradiction.

Lemma 2.4 G has no 5-cycles.

Proof. Suppose that $G$ contains the 5-cycle (see Figure 1 (8)). Then by the minimality of $G$, there is an $L$-coloring $\phi$ of $H=G-\left\{v_{i}: i \in[4]\right\}$. We want to color $e_{i}$ with a color $s_{i} \in A_{\phi}\left(e_{i}\right)$ for $i \in[9]$ such that close ones do not see each other. So we need to find $s_{i} \in A_{\phi}\left(e_{i}\right)$ for $i \in[9]$ such that $P\left(s_{1}, s_{2}, s_{3}, s_{4}, s_{5}, s_{6}, s_{7}, s_{8}, s_{9}\right) \neq 0$, where

$$
P\left(x_{1}, x_{2}, x_{3}, x_{4}, x_{5}, x_{6}, x_{7}, x_{8}, x_{9}\right)=\frac{\prod_{1 \leq k<l \leq 9}\left(x_{k}-x_{l}\right)}{\left(x_{1}-x_{9}\right)\left(x_{5}-x_{8}\right)\left(x_{3}-x_{6}\right)\left(x_{4}-x_{7}\right)} .
$$

Note that $\operatorname{deg}(P)=32,\left|A_{\phi}\left(e_{2}\right)\right| \geq 6$ and $\left|A_{\phi}\left(e_{i}\right)\right| \geq 5$ for $i \in[9]-\{2\}$. Our MATLAB codes show that $c_{P}\left(x_{1}^{4} x_{2}^{5} x_{3}^{4} x_{4}^{4} x_{5}^{3} x_{6}^{3} x_{7}^{3} x_{8}^{3} x_{9}^{3}\right)=-6$. By Lemma 1.5 , there exist $s_{i} \in A_{i}$ for $i \in[9]$ such that $P\left(s_{1}, s_{2}, s_{3}, s_{4}, s_{5}, s_{6}, s_{7}, s_{8}, s_{9}\right) \neq 0$. Note that the polynomial $P^{\prime}$ of any other 5 -cycle in $G$ is a subpolynomial of $P$, then $P \neq 0$ implies that $P^{\prime} \neq 0$ as well.

Now we are ready to prove Theorem 1.3 . 
Proof of Theorem 1.3. Let $G$ be a minimal counterexample. By Lemma 2.1-2.4, the girth of $G$ is at least six. By Euler's formula, $\sum_{v \in V(G)}(2 d(v)-6)+\sum_{f \in F(G)}(d(f)-6)=-12$. It follows that the minimum degree of $G$ is at most two, a contradiction to Lemma 2.1 that $G$ is 3-regular.

\section{Proof of Theorem 1.2}

In this section, we give a proof of Theorem 1.2 . Let $(G, L)$ be a minimal counterexample, where $|L(e)| \geq 11$ for each $e \in E(G)$. Without loss of generality, we assume $|L(e)|=11$. By Lemma 2.1 2.4. $G$ is 3 -regular and the girth of $G$ is at least six. Let $v \in V(G)$ with $N(v)=\left\{v_{1}, v_{2}, v_{3}\right\}$, and let $N\left(v_{i}\right)-\{v\}=\left\{w_{i}, w_{i}^{\prime}\right\}$ for $i \in[3]$. Then $w_{1}, w_{2}, w_{3}$ form an independent set.

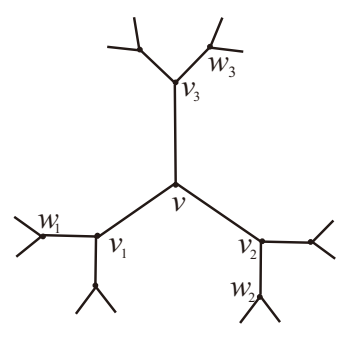

(1)

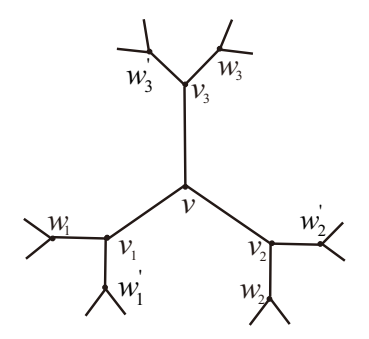

(2)

Figure 2:

Lemma 3.1 Each precoloring of $v_{1} w_{1}, v_{2} w_{2}, v_{3} w_{3}$ from their lists can be extended to an L-coloring of $H=G-v$.

Proof. Order the edges in $G$ with respect to the distance from $v$, that is, if edge $e$ precedes edge $f$, then $d(v, e) \geq d(v, f)$, where $d(v, e)$ and $d(v, f)$ are the distance from $v$ to the edges $e$ and $f$, respectively. Then the last three edges in the list are $v v_{1}, v v_{2}$ and $v v_{3}$. The edges $v_{i} w_{i}$ and $v_{i} w_{i}^{\prime}$ for $i \in[3]$ precede them. Color the edges in the list from the first to the last greedily. For each $e=x y$ in the list with $d(v, x) \geq d(v, y) \geq 1, y$ is adjacent to some vertex $z$ with $d(v, z)<d(v, y)$. So the three edges incident with $z$ are after the edge $e$ in the list. Clearly, at least two of the three edges at $z$ are not precolored, thus $e$ sees at least two uncolored edges in $G$ (Figure 3). So $e$ sees at most 10 different colors, and thus can be colored.

For $i \in[3]$, let $B_{i}=L\left(v_{i} w_{i}\right) \cup L\left(v_{i} w_{i}^{\prime}\right)$ and $L_{i}=L\left(v v_{i}\right)$. We will prove Theorem 1.2 through a series of claims.

(1) $B_{1} \cap B_{2} \cap B_{3}=\emptyset$. 


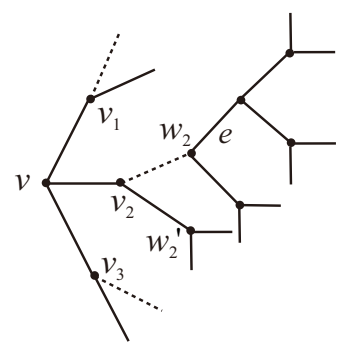

The dashed lines means the edges precolored.

Figure 3:

For otherwise, precolor $v_{i} w_{i}$ (or $v_{i} w_{i}^{\prime}$ ) with $\alpha \in B_{1} \cap B_{2} \cap B_{3}$, which can be extended to an $L$-coloring of $H$ by Lemma 3.1. Now $v v_{i}$ for $i \in[3]$ sees at most 8 different colors, so $\left|A\left(v v_{i}\right)\right| \geq 3$. So $v v_{1}, v v_{2}, v v_{3}$ can be colored in the order.

(2) For any $i, j \in[3]$ with $i \neq j, B_{i} \cap B_{j} \subseteq L_{1} \cap L_{2} \cap L_{3}$.

Suppose that for some $i, j \in[3]$ with $i \neq j$, there exists $\alpha \in\left(B_{i} \cap B_{j}\right)-\left(L_{1} \cap L_{2} \cap L_{3}\right)$. It means $\alpha$ must not belong to the one of $L_{1}, L_{2}, L_{3}$. We assume $\alpha \in\left(B_{2} \cap B_{3}\right)-L_{1}$. Without loss of generality, assume $\alpha \in L\left(v_{2} w_{2}\right) \cap L\left(v_{3} w_{3}\right)-L_{1}$. Precolor $v_{2} w_{2}, v_{3} w_{3}$ with $\alpha$ and by Lemma 3.1 . we can extend it to an $L$-coloring $\phi$ of $H$. Then $\left|A_{\phi}\left(v v_{1}\right)\right| \geq 3,\left|A_{\phi}\left(v v_{2}\right)\right| \geq 2,\left|A_{\phi}\left(v v_{3}\right)\right| \geq 2$, and we can color $v v_{2}, v v_{3}, v v_{1}$ by Lemma 1.4 .

(3) For some $i, j \in[3]$ with $i \neq j, L_{i} \cap L_{j} \neq \emptyset$.

For otherwise, in an $L$-coloring of $H$, each of $v v_{1}, v v_{2}, v v_{3}$ has an available color and the colors are distinct, so they could be colored.

We may assume that $L_{2} \cap L_{3} \neq \emptyset$.

(4) $\left|\bigcup_{i=1}^{3} B_{i}\right| \geq\left|\bigcup_{i=1}^{3} L_{i}\right|+\left|L_{1} \cap L_{2} \cap L_{3}\right|$.

By (1), $B_{1} \cap B_{2}, B_{2} \cap B_{3}, B_{3} \cap B_{1}$ are disjoint, and by (2), are subsets of $L_{1} \cap L_{2} \cap L_{3}$. So

$$
\left|\bigcup_{i=1}^{3} B_{i}\right|=\sum_{i=1}^{3}\left|B_{i}\right|-\sum_{i, j \in[3]}\left|B_{i} \cap B_{j}\right|+\left|\bigcap_{i=1}^{3} B_{i}\right| \geq \sum_{i=1}^{3}\left|B_{i}\right|-\left|\bigcap_{i=1}^{3} L_{i}\right|=33-\left|\bigcap_{i=1}^{3} L_{i}\right| .
$$

On the other hand,

$$
\left|\bigcup_{i=1}^{3} L_{i}\right|=\sum_{i=1}^{3}\left|L_{i}\right|-\sum_{i, j \in[3]}\left|L_{i} \bigcap L_{j}\right|+\left|\bigcap_{i=1}^{3} L_{i}\right| \leq 33-2\left|\bigcap_{i=1}^{3} L_{i}\right|
$$

Therefore, $\left|\bigcup_{i=1}^{3} B_{i}\right| \geq\left|\bigcup_{i=1}^{3} L_{i}\right|+\left|L_{1} \cap L_{2} \cap L_{3}\right|$.

(5) For some $i, j \in[3]$ with $i \neq j, B_{i} \bigcap B_{j} \neq \emptyset$. 
For otherwise, $\left|B_{i}\right| \geq 11$ for $i=1,2,3$ and $\left|\bigcup_{i=1}^{3} B_{i}\right| \geq 33$. Since $L_{2} \cap L_{3} \neq \emptyset$, we have $\left|\bigcup_{i=1}^{3} L_{i}\right| \leq 32$. So there exists $\alpha \in\left(B_{1} \cup B_{2} \cup B_{3}\right)-\left(L_{1} \cup L_{2} \cup L_{3}\right)$. Assume $\alpha \in L\left(v_{1} w_{1}\right) \subset B_{1}$. Since $\left|B_{2} \cup B_{3}\right| \geq 22$ and $\left|L_{2} \cup L_{3}\right| \leq 21$, there exists $\beta \in\left(B_{2} \cup B_{3}\right)-\left(L_{2} \cup L_{3}\right)$, and we may assume $\beta \in L\left(v_{2} w_{2}\right)$. Now we precolor $v_{1} w_{1}$ with $\alpha$ and $v_{2} w_{2}$ with $\beta$, and by Lemma 3.1, extend it to an $L$-coloring $\phi$ of $H$. Now $\left|A_{\phi}\left(v v_{1}\right)\right| \geq 2,\left|A_{\phi}\left(v v_{2}\right)\right| \geq 3,\left|A_{\phi}\left(v v_{3}\right)\right| \geq 3$, we can color $v v_{3}, v v_{2}, v v_{1}$ by Lemma 1.4.

By (5) and (2), $\left|L_{1} \cap L_{2} \cap L_{3}\right| \geq 1$, so by (4), there exists $\alpha \in \bigcup_{i=1}^{3} B_{i}-\bigcup_{i=1}^{3} L_{i}$. Assume $\alpha \in L\left(v_{1} w_{1}\right) \subseteq B_{1}$.

Precolor $v_{1} w_{1}$ with $\alpha$.

- $B_{2} \cap B_{3} \neq \emptyset$.

Let $\beta \in L\left(v_{2} w_{2}\right) \cap L\left(v_{3} w_{3}\right)$. Precolor $v_{2} w_{2}, v_{3} w_{3}$ with $\beta$. By Lemma 3.1, this precoloring can be extended to an $L$-coloring $\phi$ of $H$. Note that for $i \in[3],\left|A_{\phi}\left(v v_{i}\right)\right| \geq 3$, we can color $v v_{1}, v v_{2}, v v_{3}$ in the order.

- $B_{2} \cap B_{3}=\emptyset$.

Then $\left|B_{2} \cup B_{3}\right|=22-\left|B_{2} \cap B_{3}\right|=22>22-\left|L\left(e_{2}\right) \cap L\left(e_{3}\right)\right|=\left|L\left(e_{2}\right) \cup L\left(e_{3}\right)\right|$. So there exists $\beta \in\left(B_{2} \cup B_{3}\right)-\left(L_{2} \cup L_{3}\right)$. Suppose that $\beta \in L\left(v_{3} w_{3}\right)$ without loss of generality. Precolor $v_{3} w_{3}$ with $\beta$, by Lemma 3.1 , this precoloring can be extended to an $L$-coloring $\phi$ of $H$. Note that $\left|A_{\phi}\left(v v_{1}\right)\right| \geq 2,\left|A_{\phi}\left(v v_{2}\right)\right| \geq 3,\left|A_{\phi}\left(v v_{3}\right)\right| \geq 3$, and we can color $v v_{1}, v v_{2}, v v_{3}$ in the order.

\section{Final discussion}

As we mentioned in the introduction, one may try to improve our results by one, which, if true, would be optimal. But this may not be easy, especially for subcubic planar graphs.

Here is another related question. A graph is chromatic-choosable if its chromatic number equals to its list chromatic number. It is an interesting problem to find graphs that are chromaticchoosable. Zhu asked whether there exists a constant integer $k$ such that the $k$-th power $G^{k}$ is chromatic-choosable for every graph G. Kim, Kwon, and Park [10] answered this question negatively. Moreover, for any fixed $k$ they showed that there are graphs $G$ such that the value $\chi_{l}\left(G^{k}\right)-\chi\left(G^{k}\right)$ can be arbitrarily large.

We know $\chi_{s, l}^{\prime}(G)$ is the list chromatic number of the square of the line graph of $G$. Kostochka and Woodall [13] asked whether $G^{2}$ is chromatic-choosable for every graph. Kim and Park [11] 
solved the conjecture in the negative by finding a family of graphs $G$ whose squares are complete multipartite graphs with partite sets of unbounded size.

Question 4.1 Is $G^{2}$ chromatic-choosable for every line graph $G$ ?

Acknowledgement: We are very grateful for the careful reading and many helpful comments from the referees.

\section{References}

[1] N. Alon, Combinatorial Nullstellensatz, Combinatorics Probability and Computing 8 (1999) $7-29$.

[2] L. D. Andersen, The strong chromatic index of a cubic graph is at most 10, Discrete Mathematics 108 (1992) 231-252.

[3] M. Bonamy, T. Perrett, L. Postle, Colouring graphs with sparse neighbourhoods, Bounds and Applications (Submitted).

[4] H. Bruhn, F. Joos, A stronger bound for the strong chromatic index, Combinatorics, Probability and Computing 27 (2018) 21-43.

[5] D. Cranston, Strong edge-coloring of graphs with maximum degree 4 using 22 colors, Discrete Mathematics 306 (2006) 2772-2778.

[6] P. Erdős, Problems and results in combinatorial analysis and graph theory, Discrete Mathematics 72 (1988) 81-92.

[7] P. Erdős, J. Nešetřil, [Problem], in: G. Halász, V. T. Sós (Eds.), Irregularities of Partitions, Springer, Berlin, 1989, 161-349.

[8] P. Hall, On representatives of subsets, Journal of the Lond Mathematical Society 10 (1935) $26-30$.

[9] P. Horák, H. Qing, W. T. Trotter, Induced matchings in cubic graphs, Journal of Graph Theory 17 (1993) 151-160.

[10] S. J. Kim, Y. S. Kwon, B. Park, Chromatic-choosability of the power of graphs, Discrete Applied Mathematics 180 (2015) 120-125.

[11] S. J. Kim, B. Park, Counterexamples of list square coloring conjecture, Journal of Graph Theory 78 (2015) 239-247.

[12] A. V. Kostochka, X. Li, W. Ruksasakchai, M. Santana, T. Wang, G. Yu, Strong chromatic index of subcubic planar multigraphs, European Journal of Combinatorics 51 (2016) 380-397. 
[13] A. V. Kostochka, D. R. Woodall, Choosability conjectures and multicircuits, Discrete Mathematics 240 (2001) 123-143.

[14] H. Ma, Z. Miao, H. Zhu, J. Zhang, R. Luo, Strong list edge coloring of subcubic graphs, Mathematical Problems in Engineering 2013 (2013) 6 pages.

[15] M. Molloy, B. Reed, A bound on the strong chromatic index of a graph, Journal of Combinatorial Theory Series B 69 (1997) 103-109.

[16] M. Huang, M. Santana, G. Yu, Strong chromatic index of graphs with maximum degree four, submitted.

[17] H. Zhu, Z. Miao, On strong list edge coloring of subcubic graphs, Discrete Mathematics 333 (2014) 6-13.

\section{Appendix}

Note that if $P\left(x_{1}, x_{2}, \cdots, x_{m}\right)$ is a polynomial with $\operatorname{deg}(P)=n$, and $k_{1}, k_{2}, \cdots, k_{m}$ are non-negative

integers with $\sum_{i=1}^{m} k_{i}=n$. Let $c_{P}\left(x_{1}^{k_{1}} x_{2}^{k_{2}} \cdots x_{m}^{k_{m}}\right)$ be the coefficient of monomial $x_{1}^{k_{1}} x_{2}^{k_{2}} \cdots x_{m}^{k_{m}}$ in $P$. Then

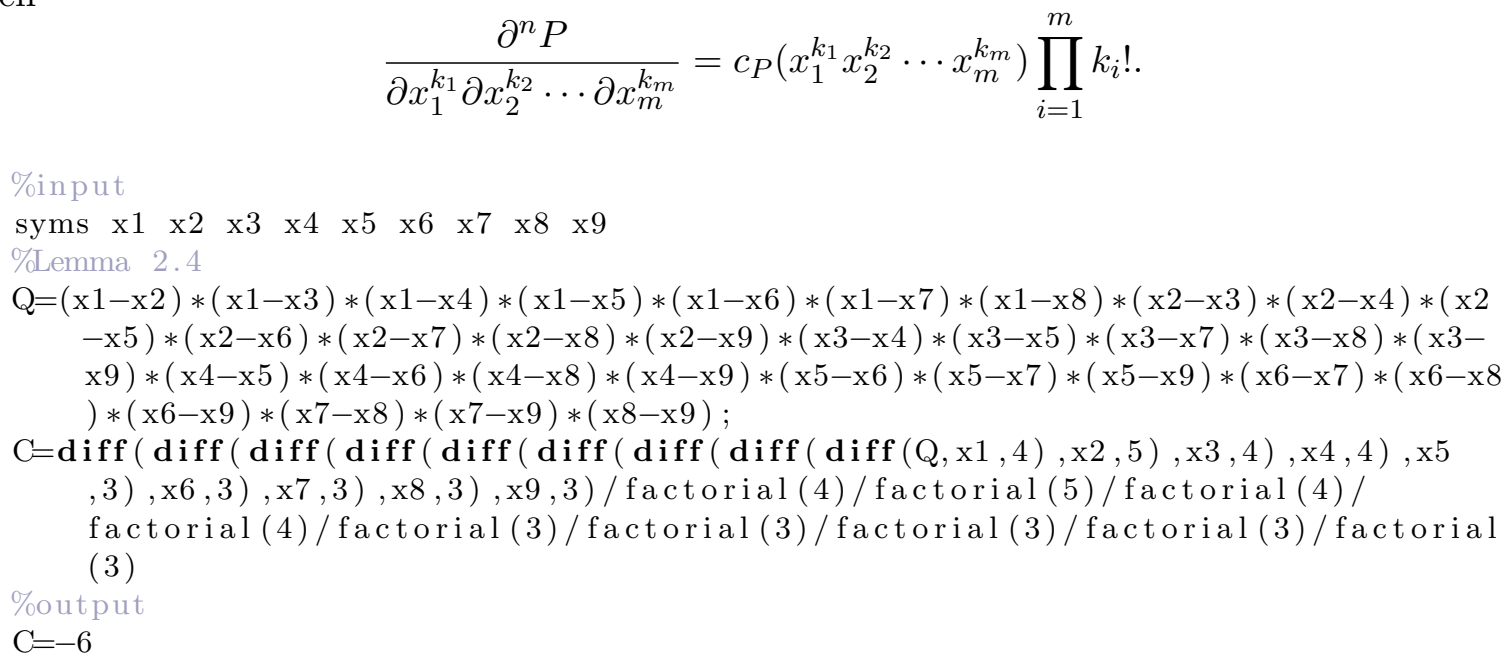$$
\frac{\partial^{n} P}{\partial x_{1}^{k_{1}} \partial x_{2}^{k_{2}} \cdots \partial x_{m}^{k_{m}}}=c_{P}\left(x_{1}^{k_{1}} x_{2}^{k_{2}} \cdots x_{m}^{k_{m}}\right) \prod_{i=1}^{m} k_{i} !
$$ 